\title{
Overview of Vascular Closure
}

The Endovascular Today annual review.

BY ZOLTAN G. TURI, MD

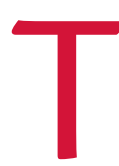
his is the seventh year that I have had the privilege of writing the annual review of vascular closure for Endovascular Today. During this period, vascular closure device (VCD) technologies have matured to the point that we are seeing mostly evolutionary changes, with at least one exception, upon which I will expand this year. Two of the original four VCDs have been replaced by several new platforms, and as of early 2009, there are five VCDs that represent the majority of the closure devices sold, along with a wide variety of topical patches. The scope of VCDs that are marketed or under development is substantial, but unlike previous years, I will not try to cover every technology; a list of devices is included on pages 34-37.

During the past year, the VCD market has recovered, paralleling the recovery of invasive cardiac and peripheral procedures in general. VCD sales are widely believed to range between $\$ 500$ million and $\$ 700$ million annually, which is an ongoing stimulus for investment resulting in a slow stream of product introductions along with takeovers of start-up companies, with new technologies being developed despite an increasingly crowded intellectual property space.

As I have each year, I will review the existing technologies, emphasizing changes in the platforms, and discuss new devices, including several under development. The classification system first introduced in Endovascular Today (Figure 1) provides a means of putting established and new VCDs in perspective and allows some anticipation of prospective strengths and weaknesses of each device being introduced. I will again take the liberty of using my annual soapbox on safety to discuss several important concepts (see sidebar, Rules of the Femoral Road) that, while being adopted by

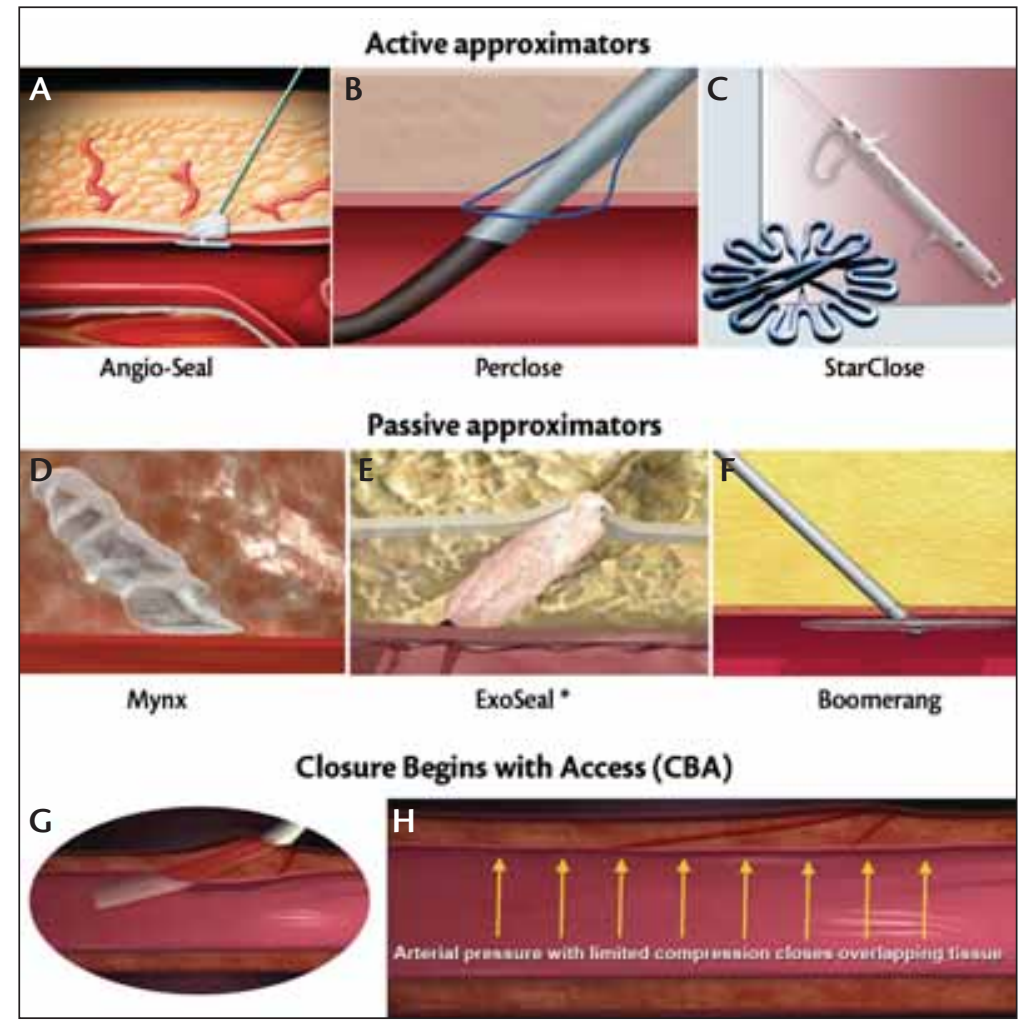

Figure 1. Vascular closure devices by mechanism of action (A-F). In the bottom row, cartoons $(\mathrm{G}, \mathrm{H})$ depict the effects of Arstasis* access and closure. On the left (G), the sheath has been placed across a shallow diagonal entry path. A small "pilot" track was used for access for the Arstasis device. On the right $(\mathrm{H})$, the sheath has been pulled from the diagonal track, which has sealed with the aid of the patient's arterial pressure. Other active approximation devices include SuperStitch (Sutura, Fountain Valley, CA), X-Site (St. Jude), and FemoSeal* (St. Jude). Other passive approximation devices include VasoSeal/On-Site (St. Jude) and Duett (Vascular Solutions). Other CBA: FISH (Femoral Introducer Sheath and Hemostasis, Morris Innovative Research, Bloomington, IN). (*Investigational device). 
more centers, need wider distribution, and cover a few of the more important articles appearing in the literature. I will also digress to the subject of radial access, still representing only a tiny percentage of cases in the US, but of increasing importance around the world.

\section{ACTIVE APPROXIMATORS - THE}

\section{"CERTAINTY" OF ACTIVE CLOSURE}

As each year for most of the past decade, Angio-Seal (St. Jude Medical, St. Paul, MN) has continued to domi- nate the market. The device sports what is generally believed to be the highest primary success rate of any VCD, in all likelihood because it features two mechanisms to achieve hemostasis: first, active approximation of the arteriotomy using a sandwich between an intraarterial resorbable anchor and an extravascular collagen plug held together by suture and second, the thrombosing effect of the collagen. It can be considered an "allweather" device-highly effective in diagnostic as well as interventional cases. The device has two potential hand-

\section{RULES OF THE FEMORAL ROAD}

I thought this would be an appropriate time to present a concatenation of the recommendations made in this series in Endovascular Today during the past 7 years. "Rules" is used here with a caveat-the recommendations that follow are based on a limited evidence base, one that is not sufficient to meet the standards of high evidence level-based guidelines, nor does failure to follow these, at least at present, violate the standard of care. Nevertheless, I believe that the evidence base will eventually catch up with what are largely common sense recommendations.

Rule No. 1. Know your anatomy. If the patient has had a previous catheterization that included femoral angiography (which should become progressively more

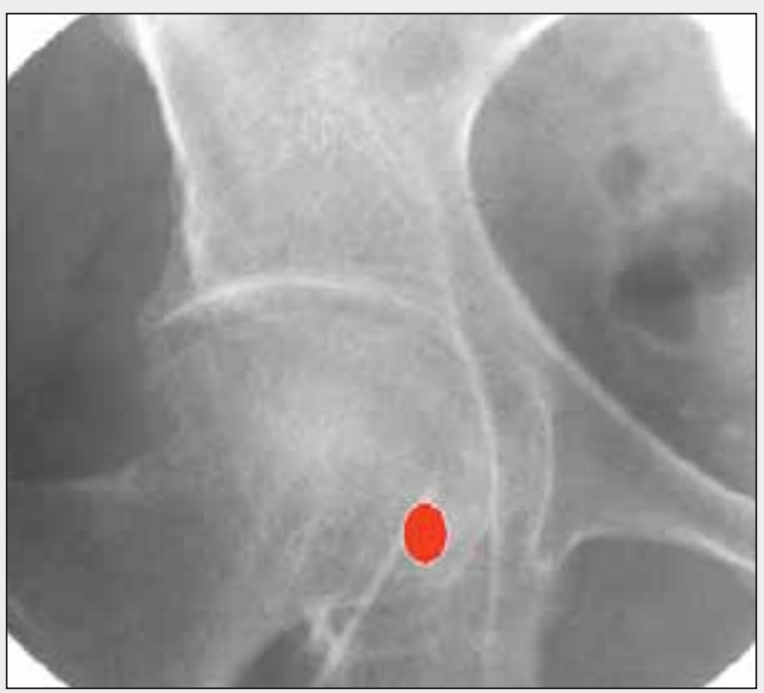

Figure 1. The target spot for vascular closure. The needle has been advanced deep into the tissue track to a point over the femoral artery, and pulsation can be felt through the needle. Prior to entering the vessel, fluoroscopy is performed to confirm a location below the centerline of the femoral head and over its medial portion. common if rule No. 4 gains acceptance), assess the films for the location of the femoral bifurcation and inferior epigastric artery with respect to the femoral head. In the case of a bifurcation at or below the bottom of the femoral head (approximately 77\% of cases), ${ }^{1}$ the target zone for arterial puncture will extend from the bottom of the femoral head to its center. Otherwise, a limited area, typically confined to a spot below the centerline of the femoral head, represents the safest choice for puncture (Figure 1).

Rule No. 2. Access using fluoroscopy and/or ultrasound guidance. To avoid the bifurcation and have the benefit of an "anvil" against which to compress the arterioto-

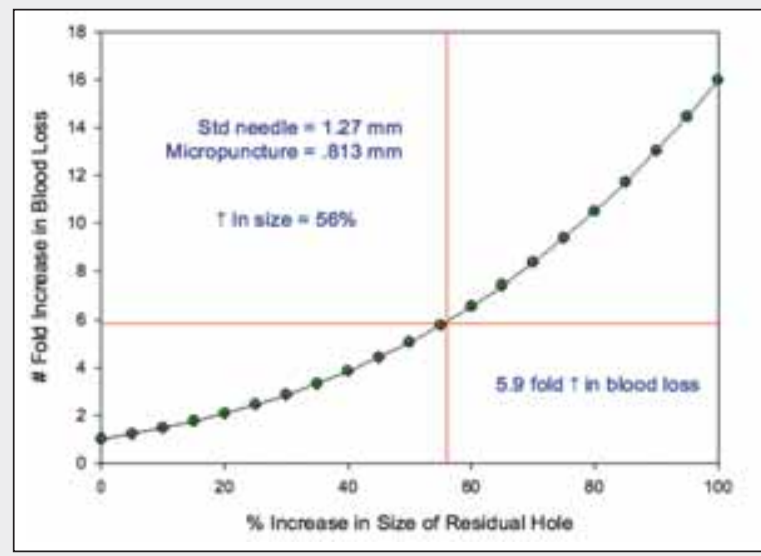

Figure 2. The relationship between the size of the needle and blood flow. A standard 18-gauge needle is approximately $56 \%$ larger than a micropuncture needle. Applying Poiseuille's Law (the resistance to flow is inversely proportion to the fourth power of the radius) and Ohm's Law (flow is inversely proportional to the resistance), the rate of blood loss from uncontrolled puncture with an access needle is nearly six times greater when an 18-gauge needle is used. 
my site, puncture over the femoral head is essential. Because the inguinal ligament rarely extends below the centerline of the femoral head, puncture over the lower half is highly desirable and readily doable if fluoroscopy is used before local anesthesia is given, and then again after the needle has been advanced subcutaneously to a point where pulsation is felt through the needle but before the tip actually enters the artery (Figure 1). Ultrasound guidance, commonly used by radiologists but still rarely by cardiologists, has significant advantages for streamlining the access process as well. I anticipate a number of relevant studies on this subject to be presented and/or published in the coming year.

Rule No. 3. Use micropuncture. A compelling case could be made for using routine micropuncture, whether the access is femoral or radial. Errant sticks, either inadvertently through the back wall of the artery or with entry into the blood vessel but inability to pass a guidewire, are quite common. Use of a standard 18-gauge needle increases the size of the hole by $56 \%$ over a 21 -gauge micropuncture needle but increases flow through the hole nearly sixfold (Figure 2). The lower flow rate and use of a straight micropuncture guidewire does require fluoroscopy to confirm that the wire has traveled up the iliac system. A number of micropuncture kits provide small-caliber, tapered, reinforced introducers that allow for atraumatic placement into some very difficult arterial and tissue anatomies.

Rule No. 4. Angiogram immediately after sheath placement. Among the risk factors for vascular access complications, three important ones can only be assessed if angiography is performed: location of puncture, size of the femoral artery, and presence of vascular disease. The optimal angle for assessing the artery and its bifurcation is usually the ipsilateral view, although the location of sheath

icaps related to properties of the technology: an anchor placed inside the artery that is, on rare occasions, obstructive, along with rare episodes of embolization and leg ischemia. The collagen inside the tissue track and a suture that extends from the arteriotomy to near the skin surface provide both a nidus and a wick for potential infection, although this occurs only in approximately $0.2 \%$ of cases. Angio-Seal has a particularly broad range of publications describing off-label use, as well as FDAapproved indications, including repuncture, although care should be taken not to re-enter the prior access track or plug site, if possible.

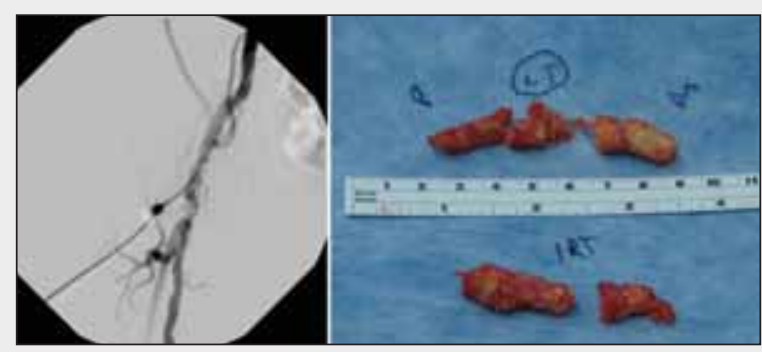

Figure 3. Routine femoral angiography can reveal the presence of severe atherosclerotic disease, making the vessel unsuitable for vascular closure device use. The tissue samples on the right were removed at endarterectomy. (Photo courtesy of James Alexander, MD).

entry is frequently better seen with the ipsilateral caudal or contralateral view. Use of half-strength contrast decreases toxicity and discomfort. Figure 3 shows a femoral artery angiogram in a patient with unsuspected common femoral artery disease.

Rule No. 5. Administer anticoagulants only after sheath angiography. The risk of all hemorrhage, and most importantly, retroperitoneal hemorrhage is increased 10to 20-fold if anticoagulants are given. In particular, if the stick is clearly high, perform those parts of the catheterization not requiring anticoagulation and save the intervention for another day.

Rule No. 6. Closure is not for the inexperienced or unsupervised. Too often, manual compression is done in the late hours by less-experienced and less-supervised individuals. One argument for VCD use is that closure takes place in the cath lab and is supervised usually by the attending performing the procedure. Vascular access and closure still represent the most common cause of cath lab complications.

Perclose and StarClose (Abbott Vascular, Santa Clara, $\mathrm{CA}$ ) remain the second most popular devices. They are particularly appealing to some users because of their parallels to the well-established surgical approaches of suturing or stapling arteriotomies. Unlike Angio-Seal, neither device resorbs, although they have smaller footprints: the Perclose suture straddles the intima inside the artery, whereas StarClose is designed to be deployed entirely outside the arterial lumen in the media. StarClose is actually a nitinol clip rather than a staple and is (in my opinion) slightly simpler to use than Perclose. In general classification terms, both feature active approximation but no 
thrombosing agent and provide what, in theory, should be less of a nidus for infection (although a difference in infection rate between Angio-Seal and StarClose has not been investigated sufficiently, and the literature has generally suggested no difference between Angio-Seal and Perclose). Both devices have some predisposition to tissue track oozing after intervention in fully anticoagulated patients, although several techniques (discussed in this space in previous years) can ameliorate or prevent this. Both devices lend themselves well to immediate repuncture, although neither has a specific FDA approval for this indication.

In the past year, Angio-Seal released its Evolution platform, which improves on the VIP version in several ways, most notably by automating the tamping down of the collagen. This allows for standardization of the force used to deliver the plug and in general, improves consistency across users. StarClose released a new platform, the SE, that also consolidates portions of the delivery process, and in addition, it provides for an enhanced release mechanism in the event that the clip does not travel through the tissue track all the way to the arteriotomy, an uncommon phenomenon most likely to occur in patients with dense scar tissue, usually from multiple previous femoral artery accesses.

\section{PASSIVE APPROXIMATORS- \\ RISE OF THE MYNX}

With an elegant deployment mechanism and excellent patient tolerance, the Mynx device (AccessClosure, Mountain View, CA) has gained significant, although still relatively modest, market share. Although conceptually similar to VasoSeal (St. Jude) and Duett (Vascular Solutions, Minneapolis, MN), two passive closure devices that have only a small remaining presence in the vascular closure world, the Mynx differs from both in several ways. First, it incorporates a sealing agent (polyethylene glycol) rather than a thrombosing agent. Second, the Mynx is introduced through the same sheath that was used for the catheterization, unlike the previously mentioned VCDs (Duett does use the procedure sheath).

These differences are potentially important. First, one could postulate that the accidental intra-arterial introduction of small amounts of sealing rather than thrombosing agent would be less likely to cause vascular thrombosis, although rare occlusion has now been reported. On the other hand, sealing agents might potentially be less effective at preventing bleeding or tissue track oozing, particularly in fully anticoagulated patients. Unfortunately, the need for scientific comparison of the efficacy and safety profile of sealing and thrombosing agents remains unmet. Second, the potential advantages of using the existing sheath for the introduction of a VCD are multiple: first, avoiding reintroduction of a device through the tissue track may decrease one of the risk factors for infection (but Mynx does still leave a foreign body in the tissue track); second, it avoids upsizing of the track at the end of the catheterization, a potential stimulus to oozing. Neither of these hypotheses has been formally tested, and as of yet, there is insufficient clinical evidence available to perform formal comparisons.

Similar to the Mynx is the ExoSeal (Cordis Corporation, Warren, NJ), which shares several characteristics: it also uses a sealant (polyglycolic acid), deployed through the procedural sheath, and also has an elegant deployment mechanism. Unlike the Mynx, the ExoSeal is not yet FDA approved. Important issues to address are the rate of resorption of polyethylene glycol versus polyglycolic acid versus collagen, as well as the degree of inflammation and fibrosis caused by each technology. Most importantly, the two VCDs that largely failed to gather market share were both passive closure devices. However, both VasoSeal and the Duett had secular issues, in part related to the thrombogenicity of those devices, as well as physical characteristics of the thrombosing agents that do not apply to the Mynx or ExoSeal. Nevertheless, they had in common with the Mynx and ExoSeal the passive closure nature of their approach to hemostasis and thus raise the possibility (demonstrated with a reasonable evidence base for VasoSeal) that the failure rate, most notably in fully anticoagulated patients, may turn out to be higher than with active approximation-type devices. Whether this will apply to the Mynx or ExoSeal is unknown. At least partly balancing this theoretical concern is the fact that passive closure devices by their nature do not leave foreign material inside the artery lumen or wall.

Finally, the Boomerang (Cardiva Medical, Mountain View, CA) is an alternative "no-footprint" device, introduced through the procedure sheath, which relies on compression of the arteriotomy from inside the vessel with a nitinol disc that is collapsed and pulled through the fresh hemostasis clot formed outside the artery. Because of its design, manual compression is required after hemostasis is otherwise achieved because the collapsed disc, reduced to the profile of an 18-gauge needle, needs to be retracted through the arteriotomy. The Catalyst version exposes the tissue track to topical agents that theoretically help promote hemostasis and prevent oozing.

\section{ARSTASIS-IN A CLASS BY ITSELF}

The most truly novel VCD is literally in a class by itself, and in some respects cannot be classified simply as a closure device. Arstasis is an access technique that lends itself to hemostasis facilitated by physiology and the laws of physics. It creates a tissue track that slants across the arterial wall in a diagonal (Figure 1), thus facilitating closure 
when the sheath is pulled, utilizing the patient's arterial pressure, as well as hydraulic principles, to seal the access track. Several hundred patients have now undergone Arstasis access, with the reported success rate in the 95\% range. Based on preliminary data presented at the TCT meeting in 2008, early experience with Arstatis access demonstrated that average hemostasis times were $3 \mathrm{~min}$ utes in diagnostic cases and 6 minutes for interventions, the latter despite ACTs in excess of 300 seconds. Although the success rate is comparable to that of existing VCDs (without a randomized study it is not possible to do a true comparison, but the results claimed are generally as good as or better than the currently marketed devices), Arstasis avoids the placement of either a temporary or permanent foreign body in the artery or tissue track at the end of the procedure. The no-foreign body-introduced/no-footprintleft-behind approach has great potential advantages because at least two of the main sources of VCD-related complications, obstruction and infection, are the result of introducing the various anchors, plugs, sutures, and clips associated with the other VCDs. However, the concept is novel enough, and the experience is still relatively small enough that the true applicability and limitations of Arstasis remain to be seen.

\section{THE BUSINESS OF VASCULAR CLOSURE}

Two companies were purchased during the previous year, resulting in implications for the VCD world. St. Jude purchased Datascope's (Montvale, NJ) vascular closure product line, which included VasoSeal/On-Site and the $\mathrm{X}$-Site, the former being the original vascular closure device introduced in the early 1990s and the active marketing of which was discontinued in late 2006. The latter is a suturebased closure device that saw limited release. St. Jude also purchased Radi Medical (Uppsala, Sweden), which gives the company the venerable FemoStop, a mainstay of assisted femoral compression, as well as the FemoSeal VCD. FemoSeal is a sandwich-type active approximator that uses a resorbable anchor inside the artery and a resorbable cap outside the vessel and is marketed in Europe. Its mechanism of action is similar to that of AngioSeal in how it achieves active approximation, but it does not incorporate a thrombosing agent inside the tissue track.

\section{RADIAL ACCESS}

This would seem to be the year to bring radial access into our annual vascular access and closure review. Although the radial approach for diagnostic and interventional cardiac catheterization is dominant in some parts of the world, particularly Europe, it remains an uncommon alternative in the US. In a review of the ACC-NCDR data- base, Rao and colleagues ${ }^{2}$ from Duke examined statistics from 2004 to 2007 and found that fewer than 8,000 of more than 593,000 coronary interventions involved radial access, a total of only $1.3 \%$. The reasons for the low rate are elusive, given that the technology has been available and widely adopted elsewhere over the past decade and a half. A number of prospective randomized trials have compared radial and femoral access, and the results have been largely concordant: radial access is safer, albeit with a lower success rate that is highly dependent on an important and prolonged learning curve. In the hands of experienced practitioners, a number of complications are quite uncommon (hematoma, bleeding, infection, pseudoaneurysm). The most lethal complication associated with femoral access, retroperitoneal hemorrhage, is (obviously) vanishingly rare via the radial approach, occurring only when there is a spontaneous bleed triggered by anticoagulation, or on occasion when radial access is used to access abdominal vessels. A number of complications distinctive to radial access remain, however, including trauma to arm vessels, obstruction of the radial (usually asymptomatic if the Allen's test confirms dual hand circulation) or higher vessels, spasm of the radial or brachial arteries with difficulties in catheter manipulation and occasionally significant pain, and trauma to the subclavian and innominate circulation.

A lingering concern relates to the passage of guidewires and catheters under the right common carotid and sometimes left common carotid (especially with bovine or common innominate/left carotid origins, which occur in close to one-third of patients combined), although a greater stroke rate has not been reported. The lack of a single catheter suitable for both coronary arteries (and left ventricular catheterization) has been a largely unsolved engineering problem for both femoral and radial access; however, the need to introduce multiple catheters is usually not a significant handicap for femoral access. With radial access, an exchange technique is commonly used that requires leaving a wire exposed under the carotids. Occasionally, the exchange through small arm vessels triggers significant spasm. A number of "one-type-serves-all" catheters have been introduced to address this, but in my experience, this sometimes means "one type serves none" because they all require some compromise in design. I have been performing radial catheterization since the mid-1990s, despite my interest in femoral access and closure, and continue to perform a portion of my coronary procedures via the radial route because several new technologies have made this approach more appealing. These technologies include improved sheaths with low coefficient of friction specifically designed to be introduced 
with a micropuncture technique and improved compression devices that rarely trigger ischemia of the hand and are extremely well tolerated by patients. Although the percentage of radial interventions was increasing near the end of the period tracked by Rao and colleagues, the technique does have sufficient limitations such that for now, at least, it will continue to be a relatively small, although growing, niche technique in the US. For those interested in the radial technique, I particularly recommend the book Patel's Atlas of Transradial Intervention: the Basics. ${ }^{3}$

\section{THE LITERATURE}

As in previous years, I have chosen three articles that were of particular interest in the past year. The study by Rao and colleagues has already been discussed. Tiroch and colleagues ${ }^{4}$ at the Brigham and Women's Hospital examined their experience with 3,400 PCls and analyzed variables that were associated with their 17 cases (0.5\%) of retroperitoneal hemorrhage (RPH). This elegant study confirmed the association between high femoral puncture and RPH. It did not confirm the previously described relationship between vascular closure devices, in particular Angio-Seal, and RPH. ${ }^{5}$ Because the number of RPH cases was small, and the techniques adapted at the Brigham (many of which were first introduced in this series over the past 7 years) represent a particularly high standard of evidence-based access and closure, I remain concerned about the general applicability of these findings with regard to VCD use. I believe the high stick/VCD/RPH phenomenon is a class effect and probably not limited to any particular device, and I included a diagram in this space last year showing the postulated mechanism, mainly involving the difficulty in pushing a plug, knot, clip, or other device down to the arterial surface when layers of muscle, such as the transversus abdominis, lie between the skin and the artery as the latter dives into the retroperitoneal space. I continue to believe, based on the overall existing evidence, that all VCDs should be used with great caution in any fully anticoagulated patient with a clearly high puncture.

Finally, Fitts and colleagues ${ }^{6}$ examined data from the Eastern Maine Medical Center, retrospectively comparing the rates of arterial injury with and without fluoroscopy use for access. The data demonstrate a significant reduction in total arterial injuries, as well as pseudoaneurysms and total length of stay when fluoroscopy was used. Although there are several study limitations, their article provides welcome confirmation that adherence to rule No. 2 (see sidebar) can in fact have a positive impact on vascular access complications. Because a number of the detailed refinements for fluoroscopic-guided access that have been presented in this column in previous years were not incorporated in this study, I would postulate that even better results can be achieved.

\section{THE CRYSTAL BALL}

Where are we going with vascular closure? The continued development of percutaneous aortic valve interventions, as well as expanding abdominal and thoracic aortic stent graft use, has led to increasing interest in percutaneous large-hole closure. Perclose, using two 6-F devices for "preclosure," and Prostar XL are particularly well positioned in this respect, and there are some excellent descriptive series showing a high success rate, despite use of sheaths up to $24 \mathrm{~F}^{7}$. There is increasing interest in closure of antegrade femoral punctures, and a number of studies have described use of many of the previously mentioned devices, albeit offlabel. ${ }^{8-10}$ Similarly, there is additional interest in using VCDs off-label for the brachial approach, although the limited depth of the arteriotomy should exclude a number of devices from consideration. ${ }^{11}$ Overall, I am aware of a half dozen VCDs making their way through bench, animal, and human testing, and I expect to have a number of other devices to discuss in the next installment of this column.

Zoltan G. Turi, MD, is Director of the Cooper Vascular Center and Professor of Medicine at Robert Wood Johnson Medical School in Camden, New Jersey. He has disclosed that he receives research grant support from Abbott Vascular and St. Jude Medical, has performed consultation for Arstasis and Johnson \& Johnson, and has received lecturing honoraria from Abbott Vascular and St. Jude Medical. Dr. Turi may be reached at (856) 342-3488; turizoltan@cooperhealth.edu.

\footnotetext{
1. Schnyder G, Sawhney N, Whisenant B, et al. Common femoral artery anatomy is influenced by demographics and comorbidity: implications for cardiac and peripheral invasive studies. Cathet Cardiovasc Interv. 2001;53:289-295.

2. Rao SV, Ou FS, Wang TY, et al. Trends in the prevalence and outcomes of radial and femoral approaches to percutaneous coronary intervention; a report from the National Cardiovascular Data Registry. J Am Coll Cardiol Interv. 2008;1:379-386.

3. Patel T. Patel's Atlas of Transradial Intervention: the Basics. Seattle, WA: Sea Script Company; 2007

4. Tiroch KA, Arora N, Matheny ME, et al. Risk predictors of retroperitoneal hemorrhage following percutaneous coronary intervention. Am J Cardiol. 2008;102:1473-1476

5. Ellis SG, Bhatt D, Kapadia S, et al. Correlates and outcomes of retroperitoneal hemorrhage complicating percutaneous coronary intervention. Cathet Cardiovasc Interv. 2006;67:541-545.

6. Fitts J, Ver LP, Hofmaster P, et al. Fluoroscopy-guided femoral artery puncture reduces the risk of PCl-related vascular complications. J Interv Cardiol. 2008;21:273-278.

7. Lee WA, Brown MP, Nelson PR, et al. Total percutaneous access for endovascular aortic aneurysm repair ("Preclose" technique). J Vasc Surg. 2007;45:1095-1101.

8. Fantoni C, Medda M, Mollichelli N, et al. Clip-based arterial haemostasis after antegrade common femoral artery puncture. Int J Cardiol. 2008;128:427-429.

9. Kapoor B, Panu A, Berscheid B. Angio-seal in antegrade endovascular interventions: technical success and complications in a 55-patient series. J Endovasc Ther. 2007:14:382-386.

10. Duda SH, Wiskirchen J, Erb M, et al. Suture-mediated percutaneous closure of antegrade femoral arterial access sites in patients who have received full anticoagulation therapy. Radiology. 1999;210:47-52.

11. Puggioni A, Boesmans E, Deloose K, et al. Use of StarClose for brachial artery closure after percutaneous endovascular interventions. Vascular. 2008;16:85-90.
} 